\begin{tabular}{|c|c|c|}
\hline \multicolumn{3}{|c|}{ Table 2} \\
\hline & $\begin{array}{c}\mathrm{CF}_{4} \\
0.852 \\
4.64\end{array}$ & $\begin{array}{c}\mathrm{CCl}_{4} \\
0.901 \\
4.86\end{array}$ \\
\hline
\end{tabular}

Finally, we may add that we have also measured the coefficient of expansion and compressibility of ammonium chloride above and below the $\lambda$-point $\left(-30 \cdot 5^{\circ}\right)$. Our results lead to values of $c_{v}$ of 18.5 cal. $/$ mole at $-44.55^{\circ}$, and $18.7 \mathrm{cal} . / \mathrm{mole}$ at $-19.31^{\circ}$ and $0^{\circ}$. These figures fit in very well with those reported by Lawson ${ }^{1}$, and confirm the conclusion he reached about the nature of the transition in this substance.

A full account of this work will be published elsewhere.

$$
\begin{aligned}
& \text { J. G. Marshall } \\
& \text { K. R. Hart } \\
& \text { L. A. K. Stafeley }
\end{aligned}
$$

Inorganic Chemistry Laboratory, Oxford. May 18.

${ }^{1}$ Lawson, A. W., Phys. Rev., 57, 417 (1940).

${ }^{2}$ Wagner, E. L., and Hornig, D. F., J. Chem. Phys., 18, 296 (1950) ${ }^{3}$ Alpert, N. I., Phys. Rev., 75 (3), 398 (1949).

${ }^{4}$ Frederick, K. J., and Hildebrand, J. H.. J. Amer. Chem. Soc., 61, 1555 (1939).

\section{Solid Phase Determinations in the System Aluminium Sulphate/Sulphuric Acid/ Water using Tracer Technique}

IN the system aluminium sulphate/sulphuric acid/water, the solid phase in the range 50-60 per cent sulphuric acid has been reported to be either $\mathrm{Al}_{2}\left(\mathrm{SO}_{4}\right)_{3} .9 \mathrm{H}_{2} \mathrm{O}^{1,2}$ or $\mathrm{Al}_{2}\left(\mathrm{SO}_{4}\right)_{3} .10 \mathrm{H}_{2} \mathrm{O}^{3}$ at temperatures between $20^{\circ}$ and $60^{\circ}$, the composition of this solid being deduced by the Schreinemakers ${ }^{4}$ method of tie-line convergence. However, in view of $(a)$ the high molecular weight of aluminium sulphate compared with sulphuric acid and water, (b) the relative position in the equilateral triangle of the solubility curve, $(c)$ inaccuracy in the position of the solution and moist solid points owing to the well-known difficulty of determining sulphuric acid in presence of aluminium sulphate, the published tie-lines show equally good convergence at points representing tho 8,9 or 10 hydrates or the $1: 1$ acid sulphate with eleven to thirteen molecules of water of crystallization.

This and similar cases 5 of ambiguity may be resolved by adding to the solubility mixture a suitable in. dicator substance which remains throughout in the liquid phase. Provided this indicator does not significantly alter the phase equilibrium and can be determined accurately in trace quantities, then the composition of the moist solid phase can be corrected for the contaminating liquid phase. Radioactive casium sulphate has been found to fulfil these severe conditions very well : only 0.05 c.c. of 0.045 per cent aqueous solution of approximately $15 \mu \mathrm{C}$. activity was required in a 50 -gm. solubility mixture. This was not added until after the ternary mixture had been brought to equilibrium at $30^{\circ}$ to avoid any co-precipitation of the tracer with the solid phase. The saturated solution and moist solid phase after convenient dilution were analysed for aluminium by precipitation with 8-hydroxyquinoline, for sulphuric acid by an accurate titration procedure, while counts were carried out in Type M6 Geiger-Müller liquid counters. Self-absorption errors were negligible because the counted solutions, after dilution with an equal volume of water, gave exactly half the original count. The solid phase was shown in this way to have the composition $51 \cdot 4$ per cent $\mathrm{Al}_{2}\left(\mathrm{SO}_{4}\right)_{3}, 15 \cdot 9$ per cent $\mathrm{H}_{2} \mathrm{SO}_{4}, 32 \cdot 7$ per cent $\mathrm{H}_{2} \mathrm{O}$, and must therefore be $\mathrm{Al}_{2}\left(\mathrm{SO}_{4}\right)_{3} \cdot \mathrm{H}_{2} \mathrm{SO}_{4} \cdot 12 \mathrm{H}_{2} \mathrm{O}$. This composition has been confirmed by using trace quantities of manganous sulphate as indicator, the manganese being determined colorimetrically as permanganate. The composition in this case was $51 \cdot 5 / 15 \cdot 1 / 33 \cdot 4$. It is surprising that this acid sulphate can be isolated in the pure state by simple filtration and thorough washing with 'Analar' acetone under anhydrous conditions, the composition of the dry $50^{\circ}$ product being $51 \cdot 4 / 15 \cdot 1 / 33 \cdot 5$.

Other acid sulphates of composition $1 / 1 / 8$ (two forms) and $1 / 1 / 2$ have been encountered in this system, and full details will be published shortly elsewhere in collaboration with Prof. H. Bassett.

DUNCAN TAYLOR

Chemistry Department, RICHARD SCOTT

University of Edinburgh.

Institute of Seaweed Research, Musselburgh.

July 4.

${ }^{1}$ Henry, J. T., and King, G. B., J. Amer, Chem. Soc., 71, $1142(1949)$ 'Funaki, K., Bull. Tokyo Inst. Tech., Series B, No. 1, 66 (1950).

${ }^{3}$ Collet, H., C.R. Acad. Sci., Paris, 230, 951 (1950).

4 Schreinemakers, F. A. H., Z. phys. Chem., 11, 81 (1893). 'Passett, H., Henshall, J. H., and Sergeant, G. A., J. Chem. Soc.,
$67 *(1939)$.

\section{Electrical Conductivity and Magnetic Susceptibility of Ovalene}

OVALINE $\left(\mathrm{C}_{32} \mathrm{H}_{14}\right)$ has the most compact configuration of rings among the condensed aromatic compounds which have so far been obtained. From this point of view, it is of interest to know the electrical and magnetic properties to be attributed to the $\pi$-electrons of this molecule.

Ovalene was synthesized by the method of Clar ${ }^{1}$ with modifications, and purified by repeated sublimation in vacuo. The measurement of the electrical conductivity as well as the magnetic susceptibility was carried out with a powder specimen. The electrical conductivity $\sigma$ can be expressed as $\sigma=\sigma_{0}$ $\exp (-\Delta \varepsilon / 2 k T)$, in the same manner as for the other condensed aromatic compounds ${ }^{2}$. The values of $\sigma$ at $15^{\circ} \mathrm{C}$. and $\Delta \varepsilon$ were found to be $4.4 \times 10^{-16}$ $\mathrm{ohm}^{-1} \cdot \mathrm{cm} .^{-1}$ and $1 \cdot 13 \mathrm{eV}$. respectively. The electrical conductivity of ovalene is less than that of violanthrone or isoviolanthrone which were reported in the previous paper. In a series of measurements on a number of the condensed aromatic compounds, we have found that the electrical conductivity depends mainly on the number of $\pi$-electrons alone, and not on the configuration of the rings. On the other hand, the magnetic susceptibility is largely influenced by the shape of the molecule.

Molecular susceptibility was found to be $\chi_{M}=$ $-(353 \cdot 8+2 \cdot 8) \times 10^{-6}$, by Gouy's method. $\chi_{M}=$ $\frac{1}{3}\left(K_{1}+K_{2}+K_{3}\right)$ can be assumed, where $K_{1}$ and $K_{2}$ are the molecular susceptibilities along two directions in the plane of the rings and $K_{3}$ is that perpendicular to the plane. It can also be assumed that $K_{1}=K_{2}=$ $\Sigma \chi(\mathrm{C}=)+\Sigma \chi(\mathrm{H})$, and calculated from Pascal's constants on the basis of spherical symmetry. $H$. Shiba and G. Hazato ${ }^{3}$ have shown that it is a good approximation to put $\chi(\mathrm{C}=)=\chi(\mathrm{C})+\frac{1}{4} \lambda$ (diethylene 